\title{
Generation of 3D ultrasound biomicroscopic images: technique validation and in vivo volumetric imaging of rat lateral gastrocnemius
}

\author{
Natália Santos da Fonseca Martins, Luisa Tinoco Carneiro, Hugo de Mello Dantas, Cláudio Esperança, \\ Ricardo Guerra Marroquim, Liliam Fernandes de Oliveira, João Carlos Machado*
}

\begin{abstract}
Introduction: Ultrasound biomicroscopy (UBM) is a technique for generating high-resolution images, with frequencies from $20 \mathrm{MHz}$ to $100 \mathrm{MHz}$. For example, it has been used in animal research related to models of injury and diseases that mimic human conditions. With a three-dimensional ultrasound (3D) image system, an organ can be viewed at various angles and the volume estimated, contributing to an accurate diagnosis. This work refers to the generation of 3D-UBM images, employing a $35 \mathrm{MHz}$ ultrasound system, from multiple two-dimensional (2D) images. Phantoms were used to validate the technique and to determine its reliability of volume measurements. Additionally, the technique was used to obtain 3D images of the rat gastrocnemius muscle. Methods: Four different phantoms were used and ten acquisition sequences of 2D-images acquired for each one. Thereafter, 5 volume segmentations were performed for each acquisition sequence, resulting in 50 measured volumes for each phantom. The physical volumes of all phantoms were used to validate the technique based on the coefficient of variation (CV) and the intraclass correlation coefficient (ICC). Images of the gastrocnemius muscle were acquired and the partial volume quantified. Results: The CV and ICC confirmed the reliability of volume measurements obtained by segmentation. Moreover, cross-sectional 2D images of rat hindlimb were obtained, allowing to identify the gastrocnemius muscle and to partially quantify the muscle volume from 3D images. Conclusion: The results indicated that the technique is valid to generate 3D images and quantify the volume of a muscle compatible with the dimensions of a small animal.
\end{abstract}

Keywords: Ultrasound biomicroscopy, 3D image, phantom, gastrocnemius muscle, rat.

\section{Introduction}

Ultrasound Biomicroscopy (UBM) is a technique working with ultrasonic frequencies from $20 \mathrm{MHz}$ to $100 \mathrm{MHz}$ to generate high-resolution images reaching, at $50 \mathrm{MHz}$, a typical range resolution of $30 \mu \mathrm{m}$ in biological tissues. UBM has application and relevance in various areas of medicine and recent highlights in biology research (Alves et al., 2013; Foster et al., 2002; Petter-Puchner et al., 2014). In medicine, the most significant topics are ophthalmology, dermatology and intravascular ultrasound with high-resolution images obtained from healthy or diseased tissues without the need of an invasive procedure (Foster et al. 2000). In biology, UBM is used in animal research involving injury and disease models that mimic human conditions, for example, rat gastrocnemius and soleus muscles response to laceration (Peixinho et al., 2011) and detection of mouse colon tumor chemically induced (Alves et al., 2013).

Likewise conventional imaging ultrasound (US) is used in medicine for diagnosis, UBM applications are, in general, based on two-dimensional (2D) images. Nevertheless, 2D-images are, sometimes, inadequate in the situation that requires full dimension organ anatomy visualization (Chang et al., 2003). In these cases, US 3D-images are advantageous because they allow organ visualization through any inspection direction (Roellig et al., 2011; Walker et al., 2009). Besides, 3D-images also allow determining, for instance, an organ volume.

Reports on the application of 3D-UBM imaging are scarce. The technique was used, in vivo, to characterize the ciliary body function during accommodation (Stachs et al., 2002). According to these authors, 2D-US imaging is unable to characterize the ciliary body function during accommodation due to the accommodative process and the presence of the ciliary processes. In another 3D-UBM application, reported by Shemesh et al. (2007), high frequency imaging US system was used to examine the 3D morphology of Streptococcus mutans biofilms grown in vitro. More recently, Foster et al. (2011) have obtained 3D-UBM image of mouse embryo along the gestation and Roellig et al. (2011) also obtained rat embryo 3D-UBM image to investigate the exceptionally long gestation period of 70 days observed in the mole-rat queen. 
Another area of investigation, in which 3D-UBM imaging can provide important contributions, refers to the analysis of skeletal muscle architecture parameters, such as muscle volume (MV), fiber length (FL) and cross sectional area (CSA), in animal models for muscle stretching and muscle regeneration following an injury. These parameters, with direct influence in the muscle function (Lieber and Friden, 2000), can be used to assess muscle contracture and to determine the changes caused by surgical interventions or specific training (Fry et al., 2007; Kawakami et al., 2008). So far, no report related with high-resolution 3D-US image of the musculoskeletal system from small animals has been found in literature.

Thus, this work was implemented to test the feasibility of a 3D-UBM imaging system to generate volumetric images of the rat gastrocnemius muscle. This imaging system acquires multiple frames of 2D-UBM images for posterior 3D conversion, segmentation and volume quantification. Initially, the system was tested with phantoms, in order to validate the technique and determine its reliability on volume measurements based on three metrics: the coefficient of variation (CV), the intraclass correlation coefficient (ICC) and the typical error of measurement (TEM). Thereafter, the 3D-UBM imaging system was tested to generate volumetric image of the rat gastrocnemius lateral muscle and to determine the corresponding volume.

\section{Methods}

\section{D-UBM image generation}

\section{Principle}

The 3D-UBM images were generated by processing 2D-UBM images acquired from multiple US parallel scan planes equally spaced and employing a commercial UBM system. The phantom (or animal) to be imaged was positioned over a platform stepped-moved in a direction perpendicular to the image scan plane.

\section{D-UBM imaging system}

A UBM system is similar to the conventional US B-mode system used in medicine and the main difference is in the higher US operating frequency.

The present work used a commercial UBM system named CLI's PC-based Imaging Sub-system and developed by Capistrano Labs Incorporated (San Clemente, CA, USA). Basically, the machine has a PC-based Imaging Sub-system, which consists of a computer PCI card that comprises a complete US imaging sub-system and includes the servo controller, the US pulser/receiver, the envelope detector, the A/D (analogic to digital) converter (sample frequency at
$80 \mathrm{MHz}$ ) and the scan converter. This card is installed in the PCI-express bus of a PC-computer mainframe containing an Intel Core i3 2100 processor. This UBM equipment operates in a Windows platform and is controlled by the Ultraview Software (version 5.93; Capistrano Labs, Incorporated, San Clemente, CA, USA). It employs a US transducer, made of polyvinylidene difluoride piezoelectric membrane (PVDF) with gold metallization (C190210; Capistrano Labs, Inc., San Clemente, CA, USA), operating at $35 \mathrm{MHz}$, that is connected to the CLI 1500Ti 35-50 probe, also fabricated by Capistrano Labs. The transducer is spherically focused with focal distance of $12 \mathrm{~mm}$ and aperture of $5 \mathrm{~mm}\left(\mathrm{f}_{\text {number }}\right.$ of 2.4). With these specifications, typical lateral and axial resolutions are $100 \mu \mathrm{m}$ and $42 \mu \mathrm{m}$, respectfully (Foster et al., 2002).

The probe is mechanically driven and provides 20 degrees sector-scan images at a rate of 20 frames/second. Each 20 degrees image sector is formed by $256 \mathrm{~A}$-lines, each line containing 2048 data points.

\section{Position system}

The platform (AS-11550; VisualSonics, Toronto, Canada) carrying the specimen to be imaged is horizontally mounted over two linear stages (MFA CC; Newport, Irvine, California, USA) configured in a horizontal XZ-plane. One stage moves in a direction (Z-axis) perpendicular to the 2D-UBM vertical image plane and each stage has a total course of $25.4 \mathrm{~mm}$, a minimum step of $0.0175 \mu \mathrm{m}$ and can be moved at a maximum speed of $2.5 \mathrm{~mm} / \mathrm{s}$.

The motion of each stage is controlled by the motion controller device (ESP300, Newport, Irvine, California, USA), programed, via RS-232 interface, by the same microcomputer that contains the Capistrano's board.

A master program, written in LabVIEW (version 7.1, National Instruments, Austin, USA) language, executes a synchronized control of the 2D-UBM system and of the platform motion.

Figure 1 depicts the UBM-2D computer mainframe and the main parts (monitor, mouse, etc.), the UBM probe with attached transducer, the fixture platform and its motion control unit.

Acquisition of multiple 2D-UBM images at equally spaced planes

During the 2D-UBM image acquisition, the linear stage in the $\mathrm{X}$-axis (parallel to the image plane) remains stationary and the one in the Z-axis moves in controlled steps. After each step in the Z-axis the positioning system remains stopped for a specified time and 32 2D-UBM images are acquired and the averaged image is determined and stored in a "BS" format, which corresponds to the 256 blocks of 2048 data points consecutively grouped in one vector. 


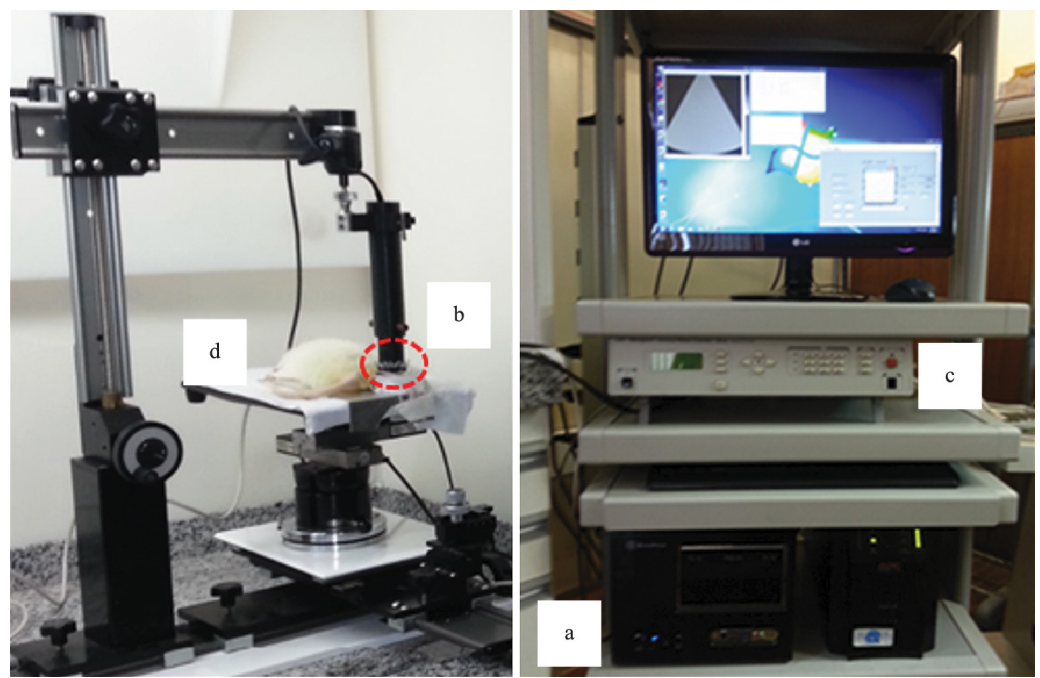

Figure 1. UBM system and animal specimen fixture: (a) computer mainframe with Capistrano UBM board, (b) UBM probe, with the part containing the transducer and in contact with the animal right hindlimb surrounded by the closed dashed-line, (c) motion control unit and (d) platform over linear stages and with an animal overlaid.

Each data point, with value between 0 and 255 , is represented in ASCII.

The operator sets the step size and the total course, and the system determines the number of corresponding steps and the maximum possible speed for the linear stage action in between consecutive steps. In the present case, a $0.2 \mathrm{~mm}$ step was chosen.

Once all the step-spaced average 2D-UBM images are acquired, the master program executes a routine, over every acquired image, for scan conversion, from polar to rectangular coordinate system, and to construct the corresponding images formed into a rectangular frame with typical dimensions of $13 \mathrm{~mm}$ $\mathrm{x} 8 \mathrm{~mm}$ for width and height, respectively. The newly formed 2D-images are stored in "jpeg" format and thereafter processed to generate de 3D-UBM image.

It typically takes about 19 minutes for the acquisition of 100 step-spaced average 2D-UBM images plus the corresponding scan conversion and storage in "jpeg" format.

\section{D-UBM image construction}

The set of 2D-images already stored in "jpeg" format is converted to "dicom" format usingthe Dicomizer (HRZ Software Services LTD, Tel-Aviv, Israel) software. Once in the dicom format, then the images are used by the TurtleSeg computer program (Medical Image Analysis Lab at Simon Fraser University, and The Biomedical Signal and Image Computing Laboratory at the University of British Columbia, Vancouver, Canada) to construct the corresponding 3D-UBM image. Subsequently, the same TurtleSeg software is used for segmentation and volume calculation.
TurtleSeg is an interactive segmentation tool originally designed for 3D medical images. A typical workflow involves the user to use 2D livewire to manually contour, using the mouse, a sparse number of different slices of the 3D image. The contouring starts by double clicking somewhere on the border of the object and using livewire to speed up the $2 \mathrm{D}$ contouring process. So, when the mouse is moved, the contour, or "livewire", automatically follows the edge of the object in real time. Left clicking will place a "seedpoint", which has the effect of anchoring the wire to that point. As seedpoints are laid along the object's boundary, TurtleSeg learns the appearance of the object being contoured, thus causing subsequent livewires to become more accurate. TurtleSeg implements a feature called spotlight, an automated system for automatically directing the user towards the next best place to provide input contours.

The full 3D segmentation is built automatically using the user-provided information. The segmented volume is calculated with the built-in measurement features of the software and the final volume value (in units of volume) is calculated through a scale calibration procedure. The complete 3D image construction, considering all the segmentation process and the volume calculation, takes about 5 minutes when performed by an experienced operator.

\section{Phantoms}

\section{Preparation}

The phantoms, appropriate for high frequency ultrasound, were prepared following the methodology described by Ryan and Foster (1997): gelatin powder 
(Type A: from porcine skin, G- 2500, Sigma Chemical Co., Missouri, USA) was dissolved in boiling water in concentrations of $15 \%$ by weight and stirred in a warm water bowl (approximately $50^{\circ} \mathrm{C}$ ) until all clumps had been dissolved. Phantom made with only gelatin is anechoic, whereas an echoic one is prepared adding silica powder (S-5631; Sigma Chemical Co., Missouri, USA) in a $2 \%$ by weight concentration. The sound speed and attenuation coefficient of the phantom containing gelatin mixed with silica powder is approximately $1549 \mathrm{~m} / \mathrm{s}$ and $2.5 \mathrm{~dB} / \mathrm{mm}$ at $35 \mathrm{MHz}$ (Ryan and Foster, 1997).

Four phantoms, with different geometries, were prepared. Each one, with an external cylindrical shape, consisted of two concentric parts; an outer cylindrical part (anechoic or echoic) involving a cylindrical or conical shape inner part (anechoic or echoic). The outer part was molded using $3 \mathrm{ml}$ (for large phantom) and $1 \mathrm{ml}$ (for small phantom) syringes (Becton Dickinson, Franklin Lakes, USA) that had their extremity, on the insertion side of the needle, sectioned transversally to the axis of syringe symmetry. The inner part was molded using $4 \mathrm{~mm}$ out diameter plastic polypropylene straw (Frascobel Disposable Plastics, Rio Grande do Sul, Brazil), or disposable $25 \mathrm{~mm} \times 0.9 \mathrm{~mm}$ and $25 \mathrm{~mm} \times 0.8 \mathrm{~mm}$ hypodermic needles (MedGoldman, Amazonas, Brazil) or even a $200 \mu$ micropipette tip in transparent polypropylene (Plast Labor, Rio de Janeiro, Brazil).

These phantoms are named $\mathrm{PH}_{\mathrm{xy}} \mathrm{T}_{\mathrm{f}}$, with "xy" representing the echogenicity ( 0 for anechoic or 1 for echoic) of outer and inner parts, respectively, "T" representing the phantom external diameter (L for large or $\mathrm{S}$ for small) and " $\mathrm{f}$ " representing the inner part format ( $\mathrm{CY}$ for cylindrical or $\mathrm{CO}$ for conical). Therefore, the four phantoms were named $\mathrm{PH}_{01} \mathrm{~L}_{\mathrm{CY}}$ (Figure 2a), $\mathrm{PH}_{10} \mathrm{~S}_{\mathrm{CY}}$ (Figure 2b), $\mathrm{PH}_{10} \mathrm{SS}_{\mathrm{CY}}$ (Figure 2c) and $\mathrm{PH}_{10} \mathrm{~L}_{\mathrm{CO}}$, (Figure 2d).
Phantom $\mathrm{PH}_{01} \mathrm{~L}_{\mathrm{CY}}$ had the outer part molded by a $3 \mathrm{ml}$ syringe (composed only of gelatin) and the inner part (gelatin mixed to silica powder) molded with the flexible straw.

Phantom $\mathrm{PH}_{10} \mathrm{~S}_{\mathrm{CY}}$ had the outer part (gelatin mixed to silica powder) molded with the $1 \mathrm{ml}$ syringe and the inner part, empty, was shaped with $0.9 \mathrm{~mm}$ diameter needle.

Phantom $\mathrm{PH}_{10} \mathrm{SS}_{\mathrm{CY}}$ was prepared similar to $\mathrm{PH}_{10} \mathrm{~S}_{\mathrm{CY}}$, but molding the inner part with a $0.8 \mathrm{~mm}$ diameter needle.

Finally, phantom $\mathrm{PH}_{10} \mathrm{~L}_{\mathrm{CO}}$, had the outer part molded with the $3 \mathrm{ml}$ syringe and the inner part, empty, molded with the micropipette tip.

The phantom preparation started with the syringe internal wall sprayed with liquid silicone oil, in order to facilitate the phantom withdrawal. Thereafter, the syringe was positioned vertically, with the bottom extremity closed by the plunger, and filled with the medium of the phantom outer part. Then, the inner part mold was concentrically inserted into the medium through the syringe top open side. Thereafter, the filled syringe was placed in the refrigerator for about 12 hours, to wait for the gelatin hardening. After this period, the inner part mold was removed and for phantom $\mathrm{PH}_{01} \mathrm{~L}_{\mathrm{CY}}$, the inner cavity was filled with gelatin mixed with silica powder. The inner cavities of the other phantoms were filled with water during phantom immersing in a water reservoir prior to imaging.

Finally, the phantoms were kept, for 5 minutes, in a $5 \%$ formalin solution (B. Herzog, Rio de Janeiro, Brazil), to increase consistency and prolong their life span up to two weeks, preventing fungi formation. Even though, all phantoms were used in the experiments immediately after the removal of formalin solution.

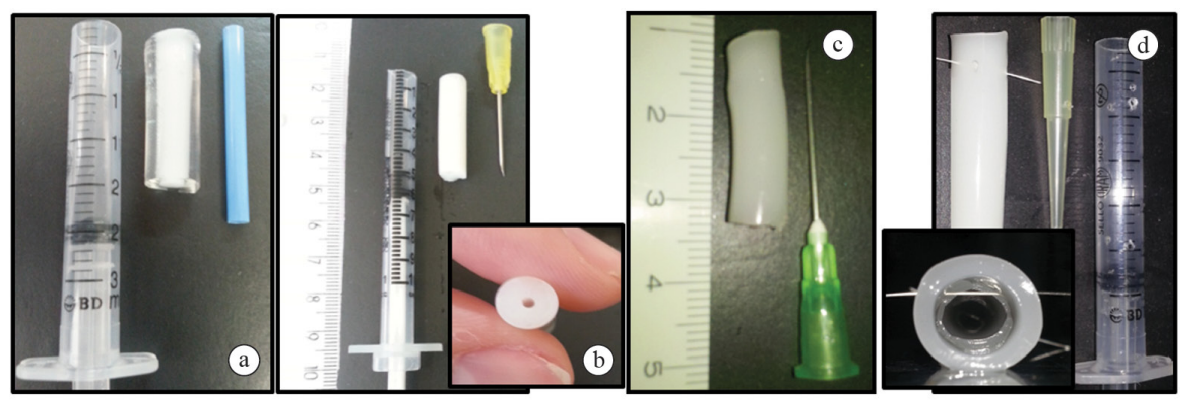

Figure 2. Main accessories used to prepare phantoms (a) $\mathrm{PH}_{01} \mathrm{~L}_{\mathrm{CY}}$ with outer part (gelatin) molded by a $3 \mathrm{ml}$ syringe and the inner part (gelatin mixed to silica powder) molded with the flexible straw, (b) $\mathrm{PH}_{10} \mathrm{~S}_{\mathrm{CY}}$ with the outer part (gelatin mixed to silica powder) molded by the $1 \mathrm{ml}$ syringe and the inner part, empty, shaped with $0.9 \mathrm{~mm}$ diameter needle, (c) $\mathrm{PH}_{10} \mathrm{SS}_{\mathrm{CY}}$ similar to $\mathrm{PH}_{10} \mathrm{~S}_{\mathrm{Cr}}$, but molding the inner part with a $0.8 \mathrm{~mm}$ diameter needle and (d) $\mathrm{PH}_{10} \mathrm{~L}_{\mathrm{CO}}$ with the outer part molded by the $3 \mathrm{ml}$ syringe and the inner part, empty, molded with the micropipette tip. 


\section{Phantom imaging}

Before starting the acquisition of the multiple 2D-UBM images of the phantom, a metal wire $(0.3 \mathrm{~mm}$ in diameter) was transversally inserted at the two ends of the phantom in order to mark the start and end points for image acquisition. Then, the phantom was fixed to a plastic structure attached to a small aluminum plate, to prevent buoyance, and immersed into distilled water contained in a plastic reservoir placed over the platform, as observed in Figure 3a. The water temperature was monitored with a thermo-hygrometer digital meter (mth-1362w; Minipa, São Paulo, Brazil) and ranged from 22 to $25^{\circ} \mathrm{C}$ during image acquisition.

In order to validate the 3D-UBM system reliability to measure volumes, through the phantom imaging, 10 acquisition sequences of multiple 2D-UBM images were performed for each phantom. The total number of frames acquired in each sequence varied from 60 to 120 , depending on the phantom length. In between each acquisition sequence of the same phantom, the wires were removed and re-introduced and the distance between them (representing the phantom length) was measured 5 times with the digital caliper (Digimess; São Paulo, Brazil), as presented in Figure 3b. The measured phantom length and diameter were used to determine its physical volume, which in turn was compared with the volume calculated from the segmented 3D-UBM images, named segmented volume. The TurtleSeg program was executed 5 times over each acquisition sequence of multiple 2D-UBM images obtained for the same phantom, and therefore, 50 (10 acquisitions $\mathrm{x} 5$ volume segmentations) volume measures were obtained for each phantom.
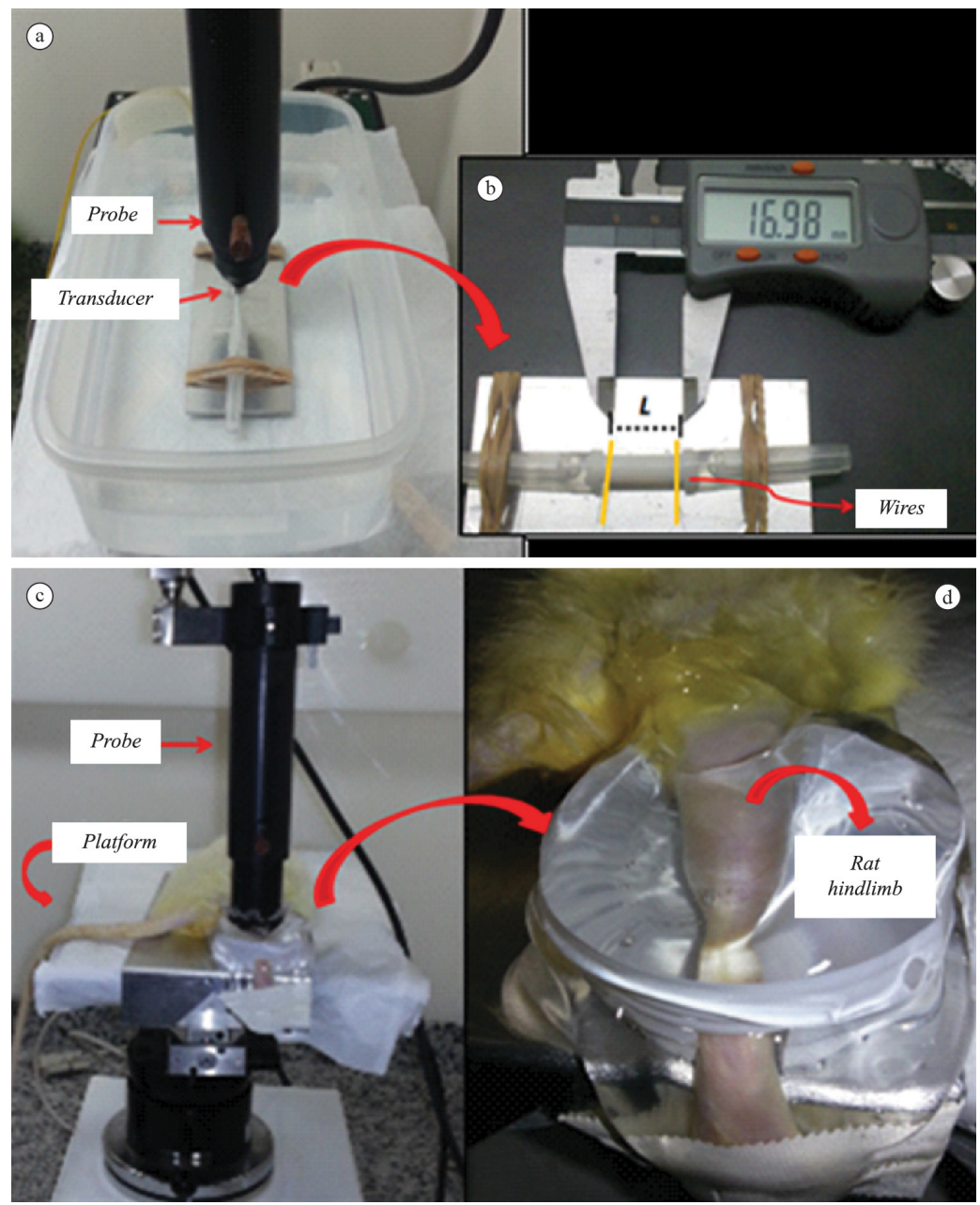

Figure 3. (a, b) Experimental set up used to acquire 2D-UBM images of the phantoms. (a) Phantom held to a plastic structure attached to a small plate of aluminum inside reservoir filled with distilled water. (b) Wires inserted at the extremities of the phantom and the measurement of distance between them with a digital caliper. (c, d) Experimental set up used to acquire 2D-UBM images of the rat hindlimb. (c) The animal positioned over the platform. (d) Detail of the rat hindlimb involved with a plastic bulkhead and covered with ultrasonic gel, with the ankle in a neutral position $\left(90^{\circ}\right)$ and internal rotation of the hip. 


\section{In vivo imaging of rat gastrocnemius muscle}

All of the experimental protocol involving the animals ( 5 Wistar rats) was performed in compliance with the recommendations of and approval of the Institutional Care and Animal Use Committee of Federal University of Rio de Janeiro (Permit number: LABCE07-09/13).

Prior to image acquisition, the rat was anesthetized with an intraperitoneal injection of ketamine $(10-15 \mathrm{mg} / \mathrm{kg})$ and xylazine $(50-75 \mathrm{mg} / \mathrm{kg})$, and had its legs tricotomized. Thereafter, the animal was placed in a prone position over the platform, as in Figure $3 \mathrm{c}$, with internal hip rotation, to ensure better alignment of muscle fibers, and the ankle in neutral position $\left(90^{\circ}\right)$, as in Figure 3d.

The animal hindlimb to be imaged was surrounded by a plastic bulkhead and fully covered with water-based ultrasonic gel (Ultrex gel; Farmativa Indústria e Comércio Ltda, Rio de Janeiro, Brazil) for acoustic coupling. The plane of 2D-UBM image was aligned perpendicularly to the longitudinal axis of the gastrocnemius muscle.

Multiple 2D-UBM images were acquired as the platform moved, perpendicularly to the image plane, in steps of $0.2 \mathrm{~mm}$. After the acquisition of multiple UBM frames in 2D, the images were analyzed and compared with the one of each leg in a rat anatomical atlas (Takamasa et al., 2001). It was possible to identify and contour the edges of the medial and lateral gastrocnemius of both legs. The total course of the linear stage was not enough to scan over the entire gastrocnemius muscle of the animals and therefore, only the partial muscle volume was possible to be determined.

\section{Validation of the $3 D-U B M$ system}

The validation of the UBM method to provide the volume of the 3D generated images was based on the comparison between segmented and physical volumes the phantoms.

\section{Statistical analysis}

The statistical analyzes over the volumetric data was conducted using the commercial statistical software SPSS 20.0 (IBM SPSS, Chicago, IL, USA).

The normal distribution of the volumetric measurements was assessed by Kolmogorov-Smirnov test. Also, the parametric one-way ANOVA for repeated measures, with Bonferroni correction, was used to verify the differences between the repeated 5 volumetric measures from the same imaging acquisition sequence and also among the 10 volumetric measures obtained during the 10 imaging acquisition sequences.
Reliability and variability of the measurements were determined by ICC or TEM and the CV, respectively. The t-test was performed to evaluate significant differences between the means of physical and segmented volumes. The significance level was set to $\mathrm{p} \leq 0.05$. The estimated error, calculated by TEM as suggested by Hopkins (2000), was used to validate the technique.

\section{Results}

\section{Segmentation and generation of $3 D$ images}

\section{Phantoms}

Typical 3D-UBM images of the phantoms are illustrated in Figures 4a-d. Also, typical segmented volumetric images of the partial lateral gastrocnemius muscle from the right hindlimb of a rat are presented, with different viewing angles, in Figure 4e.

The images from some animals did not have enough contrast between lateral gastrocnemius muscle and surround tissue in order to be processed with the TurtleSeg software and only 4 image acquisition sequences, from the partial right hindlimb of different animals, were used to calculate the partial segmented volume of the lateral gastrocnemius muscle. The measured volumes are: $3.28 \mathrm{~mm}$ (rat 2), $1.07 \mathrm{~mm}$ (rat 3), $2.44 \mathrm{~mm}$ (rat 4) and $2.04 \mathrm{~mm}$ (rat 5). The extent of the hindlimb segmented volume varied between the animals, thus the volume obtained also varied from one rat to another.

Reliability and validation of volumetric measurements for the phantoms

The segmented volumes for all the phantoms presented normal distribution. No significant difference was found applying ANOVA, with repeated measures and Bonferroni correction, for segmented and physical volumetric measures repeated five times over the same acquisition sequence. However, significant differences along the imaging acquisition sequences were found for all the phantoms. Table 1 contains the mean, standard deviation (SD), CV and ICC for the segmented volumes obtained along all the ten acquisition sequences and for each of the repeated segmentation, from first to fifth, considering all the four phantoms. Also included in Table 1 are the same metrics for segmented volumes, for all phantoms, related to five repeated segmentations and over the same acquisition sequence. The ICC for the means in Table 1 remained within the range of high reliability for all four phantoms.

In general, the $\mathrm{CV}$ for the segmented volumes obtained along the 10 acquisition sequences was higher than the $\mathrm{CV}$ for the results obtained along the repeated 
segmentations over the same acquisition sequence. The $\mathrm{CV}$ results for the phantom $\mathrm{PH}_{10} \mathrm{~L}_{\text {CO }}$ presented the highest variation interval, with a minimum value of $0.34 \%$ for repeated measurements over the same acquisition sequence and of $15.99 \%$ for the volumetric measures obtained along all the ten acquisition sequences and for one of the repeated segmentations.

Table 2 reveals the data used to compare segmented and physical volumes of the phantoms. The mean results were determined from the five measurements
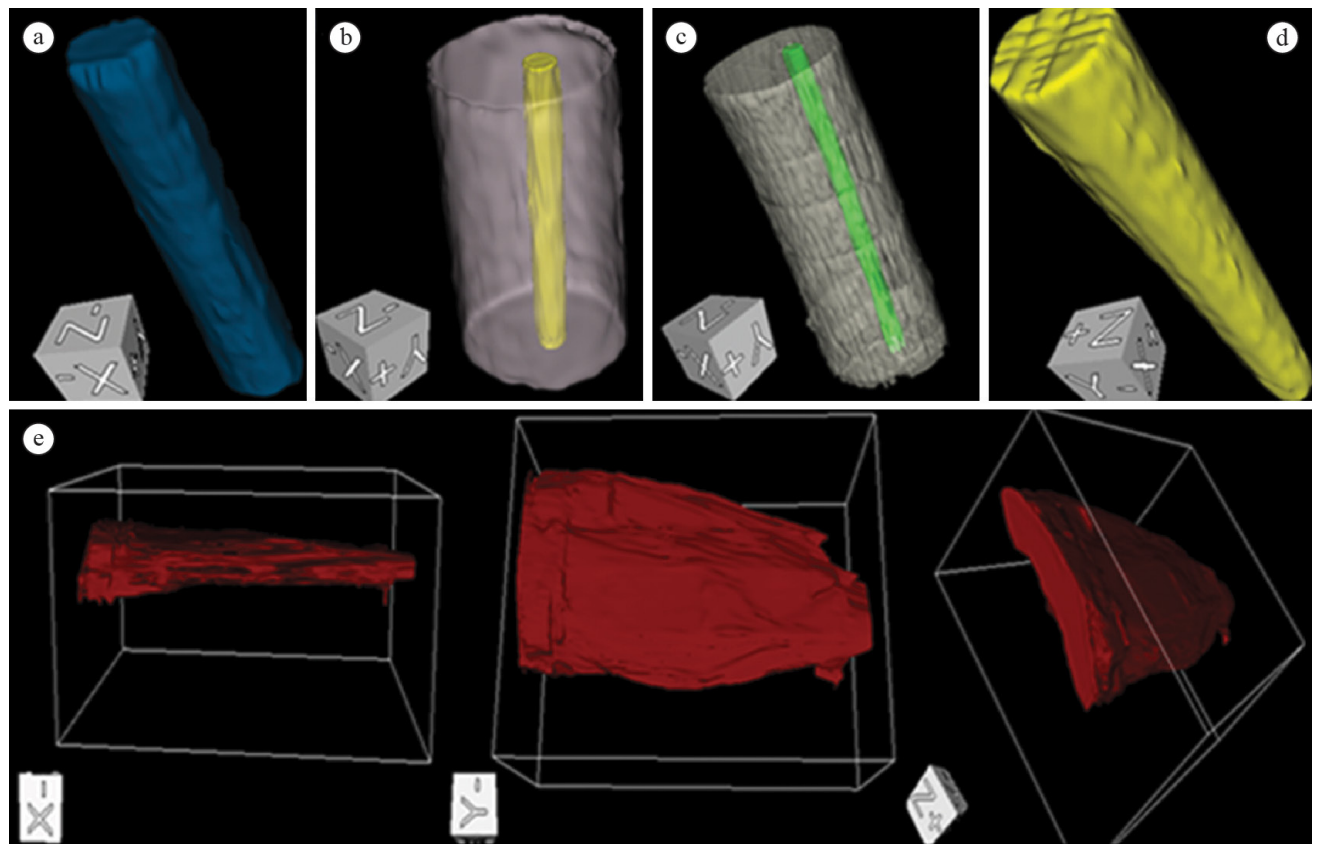

Figure 4. Typical 3D-UBM images of the four phantoms and for the rat right-side hindlimb lateral gastrocnemius at different view angles, generated after segmentation using the TurtleSeg software. (a) $\mathrm{PH}_{01} \mathrm{~L}_{\mathrm{CY}}$, (b) $\mathrm{PH}_{10} \mathrm{~S}_{\mathrm{CY}}$, (c) $\mathrm{PH}_{10} \mathrm{SS}_{\mathrm{CY}}$ (d) $\mathrm{PH}_{10} \mathrm{~L}_{\mathrm{CO}}$ and (e) lateral gastrocnemius.

Table 1. Mean, SD, CV and ICC for the segmented volumes obtained along all the ten acquisition sequences and for each of the repeated segmentation, from first to fifth, and also along the five repeated segmentations over the same acquisition sequence, for all the four phantoms.

\begin{tabular}{|c|c|c|c|c|c|c|c|c|}
\hline \multirow[b]{2}{*}{ Segmentation } & \multicolumn{2}{|l|}{$\mathrm{PH}_{01} \mathrm{~L}_{\mathrm{CY}}$} & \multicolumn{2}{|c|}{$\mathrm{PH}_{10} \mathrm{~S}_{\mathrm{CY}}$} & \multicolumn{2}{|c|}{$\mathrm{PH}_{10} \mathrm{SS}_{\mathrm{CY}}$} & \multicolumn{2}{|c|}{$\mathrm{PH}_{10} \mathrm{~L}_{\mathrm{CO}}$} \\
\hline & $\begin{array}{c}\text { Mean } \pm \text { SD } \\
\left(\mathbf{m m}^{3}\right)\end{array}$ & $\begin{array}{l}\text { CV } \\
(\%)\end{array}$ & $\begin{array}{c}\operatorname{Mean} \pm \text { SD } \\
\left(\mathbf{m m}^{3}\right)\end{array}$ & $\begin{array}{l}\text { CV } \\
(\%)\end{array}$ & $\begin{array}{c}\text { Mean } \pm \text { SD } \\
\left(\mathbf{m m}^{3}\right)\end{array}$ & $\begin{array}{l}\text { CV } \\
(\%)\end{array}$ & $\begin{array}{c}\operatorname{Mean} \pm \text { SD } \\
\quad\left(\mathbf{m m}^{3}\right)\end{array}$ & $\begin{array}{l}\text { CV } \\
(\%)\end{array}$ \\
\hline 1 & $324.20 \pm 14.96$ & 4.61 & $15.41 \pm 1.60$ & 10.41 & $15.38 \pm 1.97$ & 12.85 & $325.75 \pm 52.09$ & 15.99 \\
\hline 2 & $321.67 \pm 19.74$ & 6.13 & $15.26 \pm 1.71$ & 11.25 & $14.99 \pm 1.66$ & 11.13 & $330.84 \pm 50.54$ & 15.27 \\
\hline 3 & $322.78 \pm 18.48$ & 5.72 & $15.43 \pm 1.47$ & 9.58 & $15.00 \pm 1.88$ & 12.57 & $327.96 \pm 50.06$ & 15.26 \\
\hline 4 & $322.28 \pm 15.02$ & 4.65 & $15.39 \pm 1.42$ & 9.26 & $14.95 \pm 1.82$ & 12.23 & $328.12 \pm 51.17$ & 15.59 \\
\hline 5 & $315.77 \pm 22.44$ & 7.10 & $15.06 \pm 1.36$ & 9.04 & $15.04 \pm 1.72$ & 11.48 & $329.16 \pm 50.98$ & 14.48 \\
\hline ICC & 0.74 & & 0.90 & & 0.92 & & 0.99 & \\
\hline \multicolumn{9}{|l|}{$\begin{array}{l}\text { Acquisition } \\
\text { sequence }\end{array}$} \\
\hline 1 & $285.77 \pm 7.55$ & 2.64 & $11.32 \pm 0.36$ & 3.23 & $12.29 \pm 0.22$ & 1.88 & $241.53 \pm 1.38$ & 0.57 \\
\hline 2 & $316.61 \pm 7.12$ & 2.25 & $14.96 \pm 0.21$ & 1.40 & $13.17 \pm 0.39$ & 3.19 & $273.50 \pm 1.04$ & 0.38 \\
\hline 3 & $311.01 \pm 10.60$ & 3.41 & $15.37 \pm 0.29$ & 1.90 & $13.92 \pm 0.23$ & 1.79 & $284.32 \pm 6.29$ & 2.21 \\
\hline 4 & $323.03 \pm 4.54$ & 1.40 & $15.23 \pm 0.06$ & 0.44 & $14.11 \pm 0.18$ & 1.35 & $305.91 \pm 2.46$ & 0.80 \\
\hline 5 & $325.56 \pm 4.54$ & 4.78 & $16.69 \pm 0.43$ & 2.63 & $14.24 \pm 0.20$ & 1.47 & $323.64 \pm 1.42$ & 0.44 \\
\hline 6 & $309.78 \pm 12.19$ & 3.93 & $16.04 \pm 0.32$ & 2.02 & $15.49 \pm 0.73$ & 4.77 & $326.80 \pm 2.61$ & 0.80 \\
\hline 7 & $322.16 \pm 10.55$ & 3.27 & $17.05 \pm 0.40$ & 2.36 & $16.95 \pm 0.36$ & 2.16 & $364.51 \pm 4.38$ & 1.20 \\
\hline 8 & $346.04 \pm 8.22$ & 2.37 & $15.23 \pm 0.55$ & 0.03 & $16 ; 02 \pm 0.89$ & 5.56 & $364.68 \pm 2.84$ & 0.78 \\
\hline 9 & $334.22 \pm 6.00$ & 1.80 & $15.13 \pm 0.45$ & 3.03 & $16.15 \pm 0.51$ & 3.21 & $389.82 \pm 2.66$ & 0.68 \\
\hline 10 & $340.31 \pm 3.76$ & 1.10 & $16.07 \pm 0.17$ & 1.08 & $18.39 \pm 0.47$ & 2.59 & $408.97 \pm 1.39$ & 0.34 \\
\hline ICC & 0.93 & & 0.97 & & 0.98 & & 0.99 & \\
\hline
\end{tabular}


of segmented volumes (repeated segmentations) and of physical volumes (repeated geometric inspections) along the ten acquisition sequences. The corresponding limiting values, minimum and maximum for the mean among the ten acquisition sequences, are in Table 2, together with TEM (absolute and relative - with reference to the mean of physical volumes) and ICC, for all four phantoms. The results of TEM for phantom $\mathrm{PH}_{10} \mathrm{~S}_{\mathrm{CY}}$ also include the situation where the segmented volume of $11.32 \mathrm{~mm}^{3}$ was not considered. Even though it was not possible to track possible errors involved in the generation of segmented volume of $11.32 \mathrm{~mm}^{3}$ for phantom $\mathrm{PH}_{10} \mathrm{~S}_{\mathrm{CY}}$, this value seems to be fortunate, incompatible with all the remaining values for the segmented volume. Even though, the results for TEM and ICC for the phantom $\mathrm{PH}_{10} \mathrm{~S}_{\mathrm{CY}}$ must be considered taking into effect all the values for the measured segmented volumes (11.32 $\mathrm{mm}^{3}$ inclusive).

The t-test indicated statistical significant difference between the means of segmented and physical volumes for the phantoms $\mathrm{PH}_{10} \mathrm{SS}_{\mathrm{CY}}$ and $\mathrm{PH}_{01} \mathrm{~L}_{\mathrm{CO}}$ with the $\mathrm{p}$-value of 0.006 and 0.004 , respectively. On the other hand, the segmented and physical volumes for phantoms $\mathrm{PH}_{10} \mathrm{~S}_{\mathrm{CY}}$ and $\mathrm{PH}_{01} \mathrm{~L}_{\mathrm{CI}}$ presented no statistical significant difference, with p-value of 0.14 and 0.29 , respectively.

Regarding the technique validation, the volumetric measures for phantoms $\mathrm{PH}_{10} \mathrm{SS}_{\mathrm{CY}}$ and $\mathrm{PH}_{10} \mathrm{~L}_{\mathrm{CO}}$ presented the TEM highest relative values of $9.49 \%$ and $10.96 \%$, respectively.

The correspondence between segmented and physical volumes was also evaluated by means of the linear regression analysis. The whole set of segmented volumes, related to the four phantoms, is plotted against the corresponding physical volumes in Figure 5, together with the linear regression line fit by the method of least squares (slope $=1.09$ and intercept $=0.35 \mathrm{~mm}^{3}$ ). The corresponding Pearson correlation coefficient is $r=0.99(\mathrm{p}<0.0001)$. The slope, intercept, $r$ and $p$ for individual phantoms resulted in $1.03,-3.80 \mathrm{~mm}^{3}, 0.70$ and $0.02\left(\mathrm{PH}_{01} \mathrm{~L}_{\mathrm{CY}}\right), 2.44,-23.24 \mathrm{~mm}^{3}, 0.50$ and 0.14 $\left(\mathrm{PH}_{10} \mathrm{~S}_{\mathrm{CY}}\right), 0.55,7.98 \mathrm{~mm}^{3}, 0.23$ and $0.52\left(\mathrm{PH}_{10} \mathrm{SS}_{\mathrm{CY}}\right)$ and 4.14, $-821.47 \mathrm{~mm}^{3}, 0.90$ and $0.0006\left(\mathrm{PH}_{10} \mathrm{~L}_{\mathrm{CO}}\right)$.

\section{Discussion}

The 3D-UBM system employed in the present study was implemented mainly to obtain 3D US images of small animal organs close to the skin (maximum depth on the order of 10-12 mm). The method was tested imaging the rat gastrocnemius muscle, whose typical dimensions are about $20 \mathrm{~mm}$ length, $10 \mathrm{~mm}$ width and $3.4 \mathrm{~mm}$ thickness (Peixinho et al., 2014). The 3D image was contained in a volume with typical dimensions for length, width and thickness of $25.4 \mathrm{~mm}$, $13 \mathrm{~mm}$ and $8 \mathrm{~mm}$, respectively. These dimensions are compatible with those for the rat gastrocnemius, except for the total length that is small for larger animals. The image height depends on the transducer field of view, $0.8 \mathrm{~mm}$ in the present case. Of course, this is not an appropriate specification to generate an image with height larger than $0.8 \mathrm{~mm}$. Even so, the gastrocnemius muscle, with typical thickness of $3.4 \mathrm{~mm}$, was easily identified in the images. Moreover, this restriction can be overcome using transducers with larger $\mathrm{f}_{\text {number }}$.

A common approach to test and validate US imaging instrumentation is by means of phantoms (Barber et al., 2009; Ryan and Foster, 1997; Weller et al., 2007).

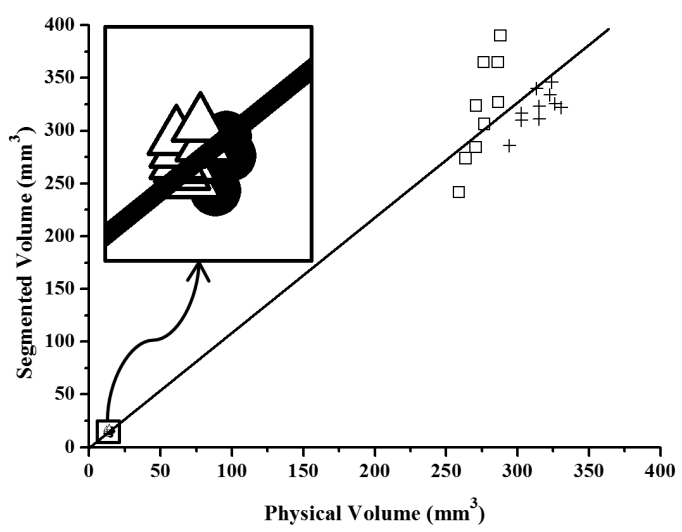

Figure 5. Linear regression line fit, by the method of least squares, to segmented versus physical volumes of the phantoms $\left(+=\mathrm{PH}_{01} \mathrm{~L}_{\mathrm{CY}}\right.$, $\left.\bullet=\mathrm{PH}_{10} \mathrm{~S}_{\mathrm{CY}}, \triangle=\mathrm{PH}_{10} \mathrm{SS}_{\mathrm{CY}}, \square=\mathrm{PH}_{10} \mathrm{~L}_{\mathrm{CO}}\right)$.

Table 2. Comparison between segment and physical volumes, including minimum and maximum results for mean, and TEM and ICC, for all four phantoms.

\begin{tabular}{|c|c|c|c|c|}
\hline & $\mathbf{P H}_{01} \mathbf{L}_{\mathrm{CY}}$ & $\mathbf{P H}_{10} \mathrm{~S}_{\mathrm{CY}}$ & $\mathrm{PH}_{10} \mathrm{SS}_{\mathrm{CY}}$ & $\mathbf{P H}_{10} \mathbf{L}_{\mathrm{CO}}$ \\
\hline \multicolumn{5}{|l|}{ Physical volume } \\
\hline mean $\left(\mathbf{m m}^{3}\right)$ & 294.21-330.50 & $15.30-16.45$ & $12.10-14.45$ & $259.18-296.01$ \\
\hline \multicolumn{5}{|c|}{ Segmented volume } \\
\hline mean $\left(\mathbf{m m}^{3}\right)$ & $285.77-346.04$ & $11.32-17.05$ & $12.29-18.39$ & $241.53-408.97$ \\
\hline \multicolumn{5}{|l|}{ TEM } \\
\hline absolute $\left(\mathrm{mm}^{3}\right)$ & 8.29 & $1.01\left(0.53^{* *}\right)$ & 1.23 & 35.99 \\
\hline relative* (\%) & 2.63 & $6.41\left(3.38^{* *}\right)$ & 9.49 & 10.96 \\
\hline ICC & 0.79 & $0.17\left(0.25^{* *}\right)$ & 0.29 & 0.54 \\
\hline
\end{tabular}

*relative to corresponding physical volume mean. **removing the outlier (volume of $11.32 \mathrm{~mm}^{3}$ ). 
Likewise, this study aimed to evaluate the 3D-UBM system conditions to generate images with compatible dimensions of rat gastrocnemius muscle and therefore its appropriateness to reproduce volumetric values similar to test objects such as phantoms. In this regard, four phantoms were prepared for this work, two of them cylindrical and with a diameter smaller than the thickness of the gastrocnemius muscle in a rat with approximately $230 \mathrm{~g}\left(\mathrm{PH}_{10} \mathrm{~S}_{\mathrm{CY}}\right.$ and $\mathrm{PH}_{10} \mathrm{SS}_{\mathrm{CY}}$ with mean length/diameter of $16.37 / 0.9 \mathrm{~mm}$ and $16.28 / 0.8 \mathrm{~mm}$, respectively). The other two phantoms were larger $\left(\mathrm{PH}_{01} \mathrm{~L}_{\mathrm{CY}}\right.$, and $\mathrm{PH}_{10} \mathrm{~L}_{\mathrm{CO}}$ with mean length/diameter of 23.75/4.1 $\mathrm{mm}$ and 24.07/2.0 $\mathrm{mm}$ (smaller diameter) and $5.6 \mathrm{~mm}$ (larger diameter), respectively).

The measured segmented volumes for the larger phantoms presented satisfactory results. Regarding the comparison of the five measures determined over the same acquisition sequence of the same phantom, the volumetric results had no significant differences, presenting ICC results within the range of greater reliability for the four phantoms (Table 1). On the other hand, the comparison of volumetric measures acquired along the 10 acquisition sequences for the same phantom leads to differences in all phantoms, indicating that phantom dimensions changed during first to the last acquisition sequence in the experiment. Nevertheless, the reliability of such measurements remained, being justified by high values of ICC and low values of $\mathrm{CV}$ values (Table 1 ). The larger phantoms, $\mathrm{PH}_{01} \mathrm{~L}_{\mathrm{CY}}$ and $\mathrm{PH}_{10} \mathrm{~L}_{\mathrm{CO}}$, had the largest variation (growth) of their volumes over the acquisitions. For phantom $\mathrm{PH}_{10} \mathrm{~L}_{\mathrm{CO}}$, the measured mean volume changed from 241.53 to $408.97 \mathrm{~mm}^{3}$ in between first and last acquisition sequences. An explanation for the phantom volume to change, as time passed, may be due to water absorption, as the phantom remained immersed in water. In general, the experiment with each phantom lasted 20 minutes and therefore the 10 acquisition sequences lasted more than 3 hours. With the phantom immersed in water throughout this time, it has changed its size, resulting in volume increase. Furthermore, the conical phantom also deformed in shape, with its axis of symmetry bending slightly at the end of the experiment.

This volume change of the phantom during the acquisitions may have influenced the corresponding TEM value of $35.99 \mathrm{~mm}^{3}$, which represents $10.96 \%$ of error, as well as the reliability since the ICC found, although within the confidence intervals, was low (0.54).

The t-test indicated significant differences (between the physical and segmented volumes) only for the phantoms $\mathrm{PH}_{01} \mathrm{~L}_{\mathrm{CO}}$, and $\mathrm{PH}_{10} \mathrm{SS}_{\mathrm{CY}}$. The reason for this result, regarding the phantom $\mathrm{PH}_{01} \mathrm{~L}_{\mathrm{CO}}$, may reside in the large variation in the physical structure of the phantom during the experiments, once the external diameters at the micropipette tip extremities did not match with corresponding diameters measured at the 2D-UBM images. Because of this fact, the diameters on the 2D-UBM images corresponding to the extremities were measured based on the software ImageJ (National Institutes of Health, Bethesda, MD, USA) and the phantom physical volume determined assuming a conical geometry. Of course, assuming the phantom conical introduces another error, once its shape changed during the experiments. This may explain why the 4 square marks, related to the largest physical volumes plotted in Figure 5, deviate considerably from the regression line.

Regarding phantom $\mathrm{PH}_{10} \mathrm{SS}_{\mathrm{CY}}$, the discrepancy between physical and segmented volumes revealed by t-test is believed due to assuming the phantom with cylindrical geometry, which in fact was not. A needle with $0.8 \mathrm{~mm}$ in diameter molded the phantom and when it was removed the bevel caused a laceration in the inner phantom wall, removing extra material and resulting in the phantom volume larger than the one calculated from a cylindrical geometry with $0.8 \mathrm{~mm}$ in diameter. Therefore, the segmented volume tended to be larger than the physical volume. Only the results of segmented volumes for the larger phantoms $\left(\mathrm{PH}_{01} \mathrm{~L}_{\mathrm{CY}}\right.$ and $\left.\mathrm{PH}_{10} \mathrm{~L}_{\mathrm{CO}}\right)$ remained within the reliability range even with low ICC values of 0.79 and 0.54 , respectively. This fact suggests that more homogeneous volumetric measurements are attained with larger phantoms.

Regarding the results for the two smaller phantoms $\left(\mathrm{PH}_{10} \mathrm{~S}_{\mathrm{CY}}\right.$, and $\left.\mathrm{PH}_{10} \mathrm{SS}_{\mathrm{CY}}\right)$, although the absolute differences were low and in the same range of values as reported by Barber et al. (2009) and Mac Gillivray et al. (2009), who compared measurements obtained by magnetic resonance imaging (MRI) with 3D-US, the corresponding values for TEM resulted in percentages of 6.41 and $9.49 \%$ respectively, and low reliability levels (ICC $=0.17$ and 0.29 , respectively) Corresponding values of ETM and ICC for phantom $\mathrm{PH}_{10} \mathrm{~S}_{\mathrm{CY}}$ changed to $3.38 \%$ and 0.25 with segmented volume of $11.32 \mathrm{~mm}^{3}$ disregarded. These results are still better than those of Delcker et al. (1999) who investigated the average deviation in the volume measurement of small muscles and found a $10 \%$ difference between the volume obtained by freehand 3D-US and by the method of water column displacement. Barber et al. (2009) considered the 3D-US system valid to measure large muscle volumes in their validation study of the same technique and confirmed the tendency to overestimate the volume measured using MRI just by 1.1\%. Barber et al. (2009) seem to have reason to believe that the errors found 
for larger structures are smaller than those found for smaller structures and may even be ignored when it comes to morphological measurements.

Currently, the literature reports studies employing methodologies similar to the one in the present work, but using US with low frequencies and applied to human research (Barber et al., 2009; Delcker et al., 1999; Infantolino et al., 2007; Weller et al., 2007).

Barber et al. (2009) proposed to validate and verify the reliability of the volume measurement for the human gastrocnemius muscle, in vivo, using 3D-US from multiple 2D scans. They compared the US results to MRI and demonstrated accuracy and repeatability of the relatively large volume muscle measurements, with a measurement error less than $2 \%$, which can be neglected to estimate muscle strength from morphological measurements. However, for small muscles, the authors emphasize that this error can be greatly increased, as in the study by Delcker et al. (1999), who found a percentage deviation of measurements ranging from $0.3 \%$ to $19.4 \%$ when compared the hand muscle volume of cadavers obtained by 3D-US and hydrostatic weighing.

Weller et al. (2007) determined the volume of the dog biceps femoris, a mid-size muscle when compared to a human one, in vivo and using a freehand 3D-US image reconstructed from multiple 2D images. In comparison with the results obtained by $\mathrm{CT}$, they concluded that the 3D-US promotes precise and accurate results, and rated the technique as an innovator in the objective to determine muscle volume in vivo. The same was observed in MacGillivray et al. (2009) study, in which 3D-US images of the femur and the rectus femoris were generated from multiple cross-sectional panorama images of the middle thigh. These authors found a mean difference of $0.53 \mathrm{~cm}^{3}$ between the volumes measured by 3D-US and MRI. This is a relative low value when compared to $3.33 \mathrm{~cm}^{3}$ of the study with dogs (Weller et al., 2007). MacGillivray et al. (2009) also observed that the in lower thigh portions, or even in subjects with member size smaller than the mean (for the elderly, for example), the difference between the volumes measured by 3D-US and MRI increased. However, the authors did not consider these differences, assessing the 3D US as an acceptable method to reproduce muscle skeletal volume. This observation seems to confirm the hypothesis of finding minor errors in larger structures.

There are also works in the literature using high frequency 2D-US images to analyze small animal biological tissues. The muscle architecture analysis of rat muscle, for example, has been performed by Peixinho et al. (2011, 2014). However, until now no reports have been found related to 3D-UBM imaging of muscular structures of these small animals.

The present study demonstrates that the 3D-UBM technique was valid for obtaining the volume of structures with dimensions similar to the rat gastrocnemius muscle. Additionally, the partial volume of this muscle was measured in 4 animals, which proved the technique ability to generate $3 \mathrm{D}$ images of the rat skeletal muscle. Future works must consider increasing the total platform course, to include the proximal muscle insertion in the 3D-UBM image.

Furthermore, the images obtained with the 3D-UBM system did not undergo any processing. As Forsberg et al. (2010) mentioned, processing techniques based in non-stationary adaptive filtering, to remove speckle and noise, may improve the image quality, keeping its structure and allowing error the reduction when measuring the volume from segmented images. The improvement for edge detection will facilitate determining the volume of a given region of interest, enabling future work such as the monitoring of tissue regeneration in rat injured skeletal muscle.

One of the major advantages for the generation of 3D-US images is the possibility to determine the volume of organs or lesion site. This has implications, for example, in treatment planning, as also in monitoring tumor response to therapy. Although MRI is considered the gold standard for direct measurement of volume and muscle length in vivo, it is costly and requires more examination time. In addition, patient sedation is need in some cases and this turns the use the 3D-US more favorable for the same purposes.

The 3D-UBM system was tested with phantoms having segmented volumes larger than the volumes measured for rat partial lateral gastrocnemius, i.e., $15 \mathrm{~mm}^{3}$ versus $1.07 \mathrm{~mm}^{3}$ (rat 3). Even so, the 3D-UBM system is still appropriate to deal with phantoms having smaller volumes, once the volume results from a combination of cross-sectional area and length. Therefore, it suffices with phantoms, likewise $\mathrm{PH}_{10} \mathrm{SS}_{\mathrm{CY}}$, being shorter. Figure 6 illustrates the correspondence between the cross-sectional areas of phantom $\mathrm{PH}_{10} \mathrm{~S}_{\mathrm{CY}}$ and of the lateral gastrocnemius at different locations along the muscle. The phantom cross-sectional area is compatible with the muscle cross-sectional area in the muscle extremity region. In addition, the phantom cross-sectional diameter is close to the muscle thickness at the mid of the muscle.

The total cost for the instrumentation used in the present work to generate 3D-UBM images amounted roughly to US $\$ 50,000.00$, without considering the cost for software implementation and the expenses to import the equipment. A commercial UMB system 

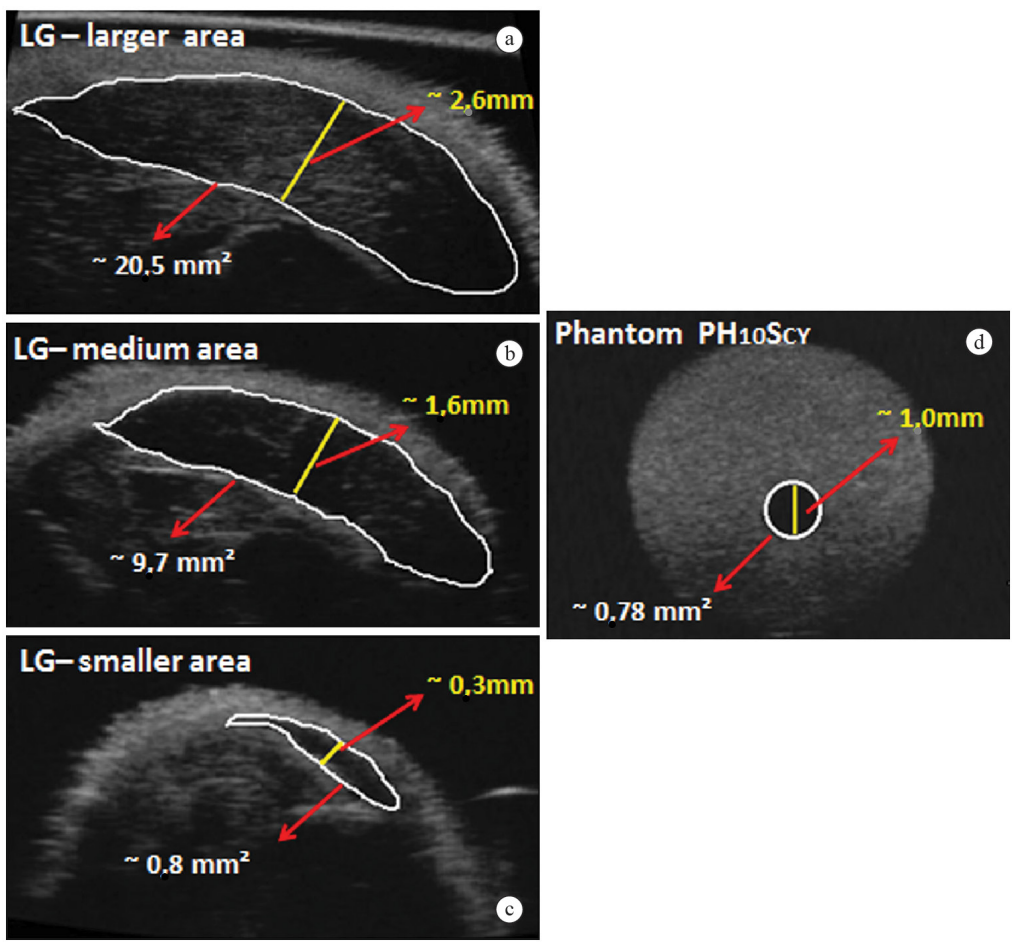

Figure 6. Typical cross-sectional areas of rat LG lateral gastrocnemius, (a) larger area (most proximal part), (b) medium area and (c) smaller area (most distal part). The area inside the highlighted white contour is in $\mathrm{mm}^{2}$. The yellow trace corresponds to the muscle thickness. (d) 2D-UBM image of phantom $\mathrm{PH}_{10} \mathrm{~S}_{\mathrm{CY}}$. The white circle area is in $\mathrm{mm}^{2}$ and the yellow trace corresponds to the diameter of the phantom.

able to generate 3D images, as well to acquire Doppler signals, roughly amounts to US400,000.00.

The results obtained in the present study indicate that the 3D-UBM system is able to generate 3D images of structures with dimensions similar to those of the tested phantoms.

The technique used in this work was able to quantify the volume of different phantoms, with a relative reliability better than $11 \%$. Even though, suitable volume determination was obtained just on larger phantoms, when comparing the means of segmented and physical volumes. It was also possible to image the rat gastrocnemius, identifying the muscle limits and determining the partial muscle volume, which justifies the originality of the work, since reports of 3D-US images from rat gastrocnemius were not found in the literature. Other studies are needed to improve the technique, although it provided images of small structures and quantified their volumes.

\section{Acknowledgements}

To the funding agencies CNPq, CAPES and FAPERJ for the financial support for the project.

\section{References}

Alves KZ, Soletti RC, Britto MA, Matos DG, Soldan M, Borges HL, Machado JC. In vivo endoluminal ultrasound biomicroscopic imaging in a mouse model of colorectal cancer. Academic Radiology 2013; 20(1):90-8. http://dx.doi. org/10.1016/j.acra.2012.07.013. PMid:22959583

Barber L, Barrett R, Lichtwark G. Validation of a freehand 3D ultrasound system for morphological measures of the medial gastrocnemius muscle. Journal of Biomechanics 2009; 42(9):1313-9. http://dx.doi.org/10.1016/j.jbiomech.2009.03.005. PMid:19375081

Chang RF, Wu WJ, Chen DR, Chen WM, Shu W, Lee JH, Jeng LB. 3-D US frame positioning using speckle decorrelation and image registration. Ultrasound in Medicine \& Biology 2003; 29(6):801-12. http://dx.doi.org/10.1016/S03015629(03)00036-X. PMid:12837496

Delcker A, Walker F, Caress J, Hunt C, Tegeler C. In vitro measurement of muscle volume with 3-dimensional ultrasound. European Journal of Ultrasound 1999; 9(2):185-90. http:// dx.doi.org/10.1016/S0929-8266(99)00023-3. PMid:10413755

Forsberg F, Berghella V, Merton DA, Rychlak K, Meiers J, Goldberg BB. Comparing image processing techniques for improved 3-dimensional ultrasound imaging. Journal of Ultrasound in Medicine 2010;29(4):615-9. PMid:20375380. 
Foster FS, Hossack J, Adamson SL. Micro-ultrasound for preclinical imaging. Interface Focus. 2011; 1(4):576-601. http://dx.doi.org/10.1098/rsfs.2011.0037. PMid:22866232

Foster FS, Pavlin CJ, Harasiewicz KA, Christopher DA, Turnbull DH. Advances in ultrasound biomicroscopy. Ultrasound in Medicine \& Biology 2000; 26(1):1-27. http:// dx.doi.org/10.1016/S0301-5629(99)00096-4. PMid:10687788

Foster FS, Zhang MY, Zhou YQ, Liu G, Mehi J, Cherin E, Harasiewicz KA, Starkoski BG, Zan L, Knapik DA, Adamson SL. A new ultrasound instrument for in vivo microimaging of mice. Ultrasound in Medicine \& Biology 2002; 28(9):116572. http://dx.doi.org/10.1016/S0301-5629(02)00567-7. PMid: 12401387

Fry NR, Gough M, McNee AE, Shortland AP. Changes in the volume and length of the medial gastrocnemius after surgical recession in children with spastic diplegic cerebral palsy. Journal of Pediatric Orthopedics 2007; 27(7):769-74. http:/ dx.doi.org/10.1097/BPO.0b013e3181558943. PMid:17878783

Hopkins WG. Measures of reliability in sports medicine and science. Sports Medicine (Auckland, N.Z.) 2000; 30(1):115. http://dx.doi.org/10.2165/00007256-200030010-00001. PMid:10907753

Infantolino BW, Gales DJ, Winter SL, Challis JH. The validity of ultrasound estimation of muscle volumes. Journal of Applied Biomechanics 2007; 23(3):213-7. PMid:18089918.

Kawakami Y, Kanehisa H, Fukunaga T. The relationship between passive ankle plantar flexion joint torque and gastrocnemius muscle and achilles tendon stiffness: implications for flexibility. The Journal of Orthopaedic and Sports Physical Therapy 2008; 38(5):269-76. http://dx.doi. org/10.2519/jospt.2008.2632. PMid: 18448880

Lieber RL, Fridén J. Functional and clinical significance of skeletal muscle architecture. Muscle \& Nerve 2000; 23(11):1647-66. http://dx.doi.org/10.1002/1097-4598(200011)23:11<1647::AIDMUS1>3.0.CO;2-M. PMid:11054744

MacGillivray TJ, Ross E, Simpson HAHRW, Greig CA. 3D freehand ultrasound for in vivo determination of human skeletal muscle volume. Ultrasound in Medicine \& Biology 2009; 35(6):928-35. http://dx.doi.org/10.1016/j. ultrasmedbio.2008.11.013. PMid:19185972

Peixinho CC, Martins NSF, de Oliveira LF, Machado JC. Structural adaptations of rat lateral gastrocnemius muscletendon complex to a chronic stretching program and their quantification based on ultrasound biomicroscopy and optical microscopic images. Clinical Biomechanics (Bristol,
Avon) 2014; 29(1):57-62. http://dx.doi.org/10.1016/j. clinbiomech.2013.11.002. PMid:24309012

Peixinho CC, Ribeiro MB, Resende CMC, Werneck-de-Castro JPS, Oliveira LF, Machado JC. Ultrasound biomicroscopy for biomechanical characterization of healthy and injured triceps surae of rats. The Journal of Experimental Biology 2011; 214(Pt 22):3880-6. http://dx.doi.org/10.1242/jeb.059808. PMid:22031753

Petter-Puchner A, Gruber-Blum S, Walder N, Fortelny RH, Redl H, Raum K. Ultrasound biomicroscopy (UBM) and scanning acoustic microscopy (SAM) for the assessment of hernia mesh integration: a comparison to standard histology in an experimental model. Hernia 2014; 18(4):579-85. http:// dx.doi.org/10.1007/s10029-013-1201-9. PMid:24346242

Roellig K, Drews B, Goeritz F, Hildebrandt TB. The long gestation of the small naked mole-rat (Heterocephalus glaber Rüppell, 1842) studied with ultrasound biomicroscopy and 3D-ultrasonography. PLoS ONE 2011; 6(3):e17744. http:// dx.doi.org/10.1371/journal.pone.0017744. PMid:21408185

Ryan LK, Foster FS. Tissue equivalent vessel phantoms for intravascular ultrasound. Ultrasound in Medicine \& Biology 1997; 23(2):261-73. http://dx.doi.org/10.1016/ S0301-5629(96)00206-2. PMid:9140183

Shemesh H, Goertz DE, van der Sluis LW, de Jong N, Wu MK, Wesselink PR. High frequency ultrasound imaging of a single-species biofilm. Journal of Dentistry 2007; 35(8):673-8. http://dx.doi.org/10.1016/j.jdent.2007.05.007. PMid:17604896

Stachs O, Martin H, KirchhoffA, Stave J, Terwee T, Guthoff R. Monitoring accommodative ciliary muscle function using three-dimensional ultrasound. Graefes Archive for Clinical and Experimental Ophthalmology 2002; 240(11):906-12. http:// dx.doi.org/10.1007/s00417-002-0551-2. PMid:12486512

Takamasa I, Hiroshi Y, Toshiyuki H. A color atlas of sectional anatomy of the rat. Japan; 2001.

Walker M 3rd, Campbell BR, Azer K, Tong C, Fang K, Cook JJ, Forrest MJ, Kempadoo K, Wright SD, Saltzman JS, MacIntyre E, Hargreaves R. A novel 3-dimensional micro-ultrasound approach to automated measurement of carotid arterial plaque volume as a biomarker for experimental atherosclerosis. Atherosclerosis 2009; 204(1):55-65. http://dx.doi.org/10.1016/j. atherosclerosis.2008.09.013. PMid:19135672

Weller R, Pfau T, Ferrari M, Griffith R, Bradford T, Wilson A. The determination of muscle volume with a freehand 3D ultrasonography system. Ultrasound in Medicine \& Biology 2007; 33(3):402-7. http://dx.doi.org/10.1016/j. ultrasmedbio.2006.08.007. PMid:17208353

\section{Authors}

Natália Santos da Fonseca Martins ${ }^{1}$, Luisa Tinoco Carneiro ${ }^{1}$, Hugo de Mello Dantas ${ }^{1}$, Cláudio Esperança ${ }^{2}$, Ricardo Guerra Marroquim $^{2}$, Liliam Fernandes de Oliveira ${ }^{1}$, João Carlos Machado ${ }^{1,3 *}$

${ }^{1}$ Programa de Engenharia Biomédica, Instituto Alberto Luiz Coimbra de Pós-Graduação e Pesquisa de Engenharia COPPE, Universidade Federal do Rio de Janeiro - UFRJ, Rio de Janeiro, RJ, Brasil.

${ }^{2}$ Programa de Engenharia de Sistemas e Computação, Instituto Alberto Luiz Coimbra de Pós-Graduação e Pesquisa de Engenharia - COPPE, Universidade Federal do Rio de Janeiro - UFRJ, Rio de Janeiro, RJ, Brasil.

${ }^{3}$ Programa de Pós-Graduação em Ciências Cirúrgicas, Faculdade de Medicina, Universidade Federal do Rio de Janeiro UFRJ, Rio de Janeiro, RJ, Brasil. 